\section{CardioRenal Medicine}

\title{
Effects of Bardoxolone Methyl on Magnesium in Patients with Type 2 Diabetes Mellitus and Chronic Kidney Disease
}

\author{
Dana V. Rizk $^{a} \quad$ Arnold L. Silva $^{b}$ Pablo E. Pergola ${ }^{c}$ Robert Toto ${ }^{d}$ \\ David G. Warnock ${ }^{a}$ Melanie P. Chin ${ }^{\mathrm{e}}$ Angie Goldsberry ${ }^{\mathrm{e}}$ \\ Megan O'Gradye Colin J. Meyer ${ }^{\mathrm{e}}$ Peter A. McCullough ${ }^{f}$ \\ a Department of Medicine, Nephrology Division, University of Alabama at Birmingham, \\ Birmingham, AL, USA; ${ }^{b}$ Boise Kidney and Hypertension Institute, Meridian, ID, USA; \\ 'Renal Associates PA, San Antonio, TX, USA; ${ }^{d}$ Internal Medicine and Clinical Science,

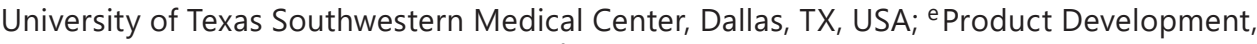

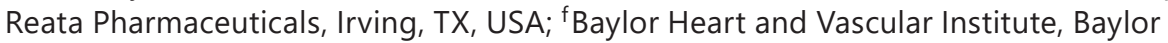 \\ Jack and Jane Hamilton Heart and Vascular Hospital, The Heart Hospital, Baylor University \\ Medical Center, Dallas, TX, USA
}

\section{Keywords}

Bardoxolone methyl - Magnesium - Chronic kidney disease

\begin{abstract}
Background: Treatment with bardoxolone methyl (Bard) in a multinational phase 3 trial, Bardoxolone Methyl Evaluation in Patients with Chronic Kidney Disease and Type 2 Diabetes (BEACON), resulted in increases in estimated glomerular filtration rate with concurrent reductions in serum magnesium. We analyzed data from several trials to characterize reductions in magnesium with Bard. Methods: BEACON randomized patients $(n=2,185)$ with type 2 diabetes (T2DM) and stage 4 chronic kidney disease (CKD) 1:1 to receive Bard (20 mg) or placebo once daily. In a separate open-label study, magnesium levels from 24-hour urine and sublingual epithelial cell samples were analyzed in patients with stage 3b-4 CKD and T2DM administered $20 \mathrm{mg}$ Bard for 56 consecutive days. Results: BEACON patients randomized to Bard experienced significant reductions in serum magnesium from baseline relative to patients randomized to placebo $(-0.17 \mathrm{mEq} / \mathrm{L}, 95 \% \mathrm{Cl}-0.18$ to $-0.60 \mathrm{mEq} / \mathrm{L} ; p<0.001)$. A separate study showed intracellular and urinary magnesium levels were unchanged with Bard treatment. Conclusions: Bard treatment results in significant decreases in serum magnesium that are not associated with changes in intracellular and urinary magnesium levels, indicating that magnesium decreases are not due to renal magnesium wasting or total body magnesium depletion. Importantly, the decreases in serum magnesium with Bard are not associated with adverse effects on QT interval.


Rizk et al.: Effects of Bardoxolone Methyl on Magnesium

\section{Introduction}

Bardoxolone methyl activates the nuclear factor E2-related factor 2 (Nrf2) pathway, which plays an important role in maintaining kidney function and structure [1-5]. Bardoxolone methyl interacts with cysteine residues on kelch-like ECH-associated protein 1 (Keap1) allowing Nrf2 translocation to the nucleus and subsequent upregulation of a multitude of antioxidant genes $[1,5,6]$. Additionally, the structure and activity profile of bardoxolone methyl resemble those of the cyclopentenone prostaglandins, endogenous Nrf2 activators that exert anti-inflammatory effects by inhibiting the proinflammatory nuclear factor $\kappa \mathrm{B}$ pathway [7-10].

Preclinical studies have shown that bardoxolone methyl and close analogs have beneficial activity in animal models of kidney disease, including amelioration of murine ischemic acute kidney injury [11], attenuation of kidney interstitial inflammation and fibrosis in mice with proteinuria induced by protein overload $[12,13]$, and protection against fibrosis in a $5 / 6$ nephrectomy model of chronic kidney disease (CKD) [14].

Improvements in kidney function, assessed using measured inulin clearance, measured creatinine clearance, and estimated glomerular filtration rate (eGFR), have also been observed consistently with bardoxolone methyl treatment in several clinical trials [15-18]. The largest of these was a multinational, randomized, double-blind, placebo-controlled phase 3 outcomes trial, which enrolled 2,185 patients with type 2 diabetes mellitus (T2DM) and CKD stage 4 (Bardoxolone Methyl Evaluation in Patients with Chronic Kidney Disease and Type 2 Diabetes: The Occurrence of Renal Events [BEACON]) [17]. The BEACON trial was terminated early because of safety concerns, largely driven by a significant increase in heart failure events within the first 4 weeks of exposure. The cause of these events was subsequently determined to be due to acute fluid retention without evidence of direct cardiotoxicity and occurred in a subset of patients with identifiable risk factors (elevated baseline B-type natriuretic peptide and prior history of heart failure) $[19,20]$, which allowed for exclusion of at-risk patients in subsequent trials.

Despite the early termination of BEACON, patients randomized to bardoxolone methyl in BEACON experienced significantly improved kidney function. Lower serum creatinine concentrations (corresponding to higher eGFRs), along with lower serum concentrations of urea nitrogen, uric acid, and phosphorus were observed. In concert with improved kidney function, reductions in serum magnesium were also observed in bardoxolone methyl-treated patients, and hypomagnesemia was reported as an adverse event in $21 \%$ of patients randomized to bardoxolone methyl compared with $6 \%$ of placebo patients [17]. As a result, we performed analyses from BEACON and an additional pharmacodynamic study, which was conducted in parallel with BEACON, to further characterize reductions in serum magnesium induced by bardoxolone methyl treatment.

\section{Methods}

BEACON Study

The BEACON trial (NCT01351675) was a phase 3, randomized, double-blind, parallel-group, international, multicenter trial of once-daily administration of bardoxolone methyl (20 $\mathrm{mg}$ ), as compared with placebo. Previous publications describe the BEACON trial design in detail $[17,21]$. Briefly, BEACON enrolled adults with T2DM and stage $4 \mathrm{CKD}$, corresponding to an eGFR of 15 to $<30 \mathrm{~mL} / \mathrm{min} / 1.73 \mathrm{~m}^{2}$. Patients were randomized 1:1 to once-daily administration of bardoxolone methyl or placebo. Patients received background conventional therapy that included inhibitors of the renin-angiotensin-aldosterone system, insulin or other hypoglycemic agents, and, when appropriate, other cardiovascular medications. 
eGFR was calculated using serum creatinine and the 4-variable Modification of Diet in Renal Disease (MDRD) study equation. eGFR and clinical chemistries (including serum magnesium) were assessed every 4 weeks through week 12, followed by assessments every 8 weeks thereafter. Electrocardiograms were collected every 24 weeks.

The primary composite endpoint of the trial was the time to first event in the composite outcome defined as end-stage kidney disease (need for maintenance dialysis, kidney transplantation, or renal death) or cardiovascular death. Secondary efficacy outcomes included the change in eGFR, time to first hospitalization for heart failure or death due to heart failure, and time to first event of a composite consisting of non-fatal myocardial infarction, non-fatal stroke, hospitalization for heart failure, or cardiovascular death.

The BEACON population was analyzed in accordance with the intention-to-treat principle. Longitudinal analyses of serum magnesium were used to compare mean changes in serum magnesium between the bardoxolone methyl and placebo groups. As previously described, mixed-effects regression used postbaseline serum magnesium as the response variable; treatment group, time, the interaction of treatment group with time, interaction of serum magnesium with time; and continuous covariates (serum magnesium at baseline, baseline eGFR, and urinary albumin-to-creatinine ratio) [17]. Longitudinal analyses were performed with the use of SAS software, version 9.3 (SAS Institute). In addition, summary statistics were to compare changes in serum magnesium QT interval corrected by Fridericia's formula (QTcF) in patients randomized to bardoxolone methyl or placebo. One-sample (paired) $t$ tests were used for comparing mean changes to zero and two-sample $t$ tests were used to compare the difference in means between bardoxolone methyl and placebo groups.

\section{Pharmacodynamic Study}

To further characterize the pharmacodynamic effects of bardoxolone methyl, 24 patients with CKD stage 3b-4 (eGFR 15-45 mL/min/1.73 $\mathrm{m}^{2}$ ) and type 2 diabetes were enrolled in a multicenter, open-label study (NCT01549769) and were administered $20 \mathrm{mg}$ bardoxolone methyl once daily for 56 consecutive days. Patients returned for clinical and laboratory evaluations on days 28 and 56 (end of treatment), and posttreatment follow-up on days 60 and 84 . The primary objective of the study was to assess the pharmacokinetic profile of bardoxolone methyl in patients with CKD and type 2 diabetes. Additional objectives included assessing pharmacodynamic responses to bardoxolone methyl, including fractional excretion of magnesium and other electrolytes. The study was also halted on October 18, 2012 after premature termination of BEACON.

During each visit, vital signs, electrocardiograms, concomitant medication list, and adverse events were documented. Blood, urine, and sublingual epithelial cell samples were collected at different time points during the study. Patients were also asked to collect 24-hour urine samples during the screening and on days $1,28,56$, and 84 of the study. eGFR was calculated using the MDRD equation. Sublingual epithelial cell specimens were used to determine intracellular magnesium levels using a previously validated method [22]. Following study termination, patients were asked to stop taking bardoxolone methyl; however, they were asked to complete two additional study visits approximately 4 weeks apart after stopping bardoxolone methyl to ensure patient safety.

Observed, change-from-baseline, and percent-change-from-baseline values for clinical laboratory values and 24-hour excretion variables (including fractional excretions) were summarized descriptively at all scheduled sampling points for all patients. All available data from patients receiving at least 1 dose of study drug were included in the analyses. No adjustments or imputations were utilized for missing values or for patients who withdrew prior to completing the study. Paired $t$ tests were used to compare differences in baseline and post-baseline values of selected clinical laboratory parameters.

All studies were approved by Institutional Review Boards at participating sites and were conducted in accordance with the Declaration of Helsinki. All patients or subjects provided written informed consent.

\section{Results}

Patient Population

BEACON

From June 2011 through September 2012, a total of 2,185 patients were randomized in BEACON to receive either bardoxolone methyl $(n=1,088)$ or placebo $(n=1,097)$ [17]. Previous 
Table 1. Select demographics and baseline characteristics from the BEACON and pharmacodynamic studies

\begin{tabular}{|c|c|c|c|}
\hline & \multicolumn{2}{|c|}{ BEACON study ${ }^{\mathrm{a}}$} & \multirow{2}{*}{$\begin{array}{l}\text { Pharmaco- } \\
\text { dynamic } \\
\text { study }(n=24)\end{array}$} \\
\hline & $\begin{array}{l}\text { placebo } \\
(n=1,097)\end{array}$ & $\begin{array}{l}\text { bardoxolone } \\
\text { methyl }(n=1,088)\end{array}$ & \\
\hline Age, years (mean \pm SD) & $68 \pm 9$ & $69 \pm 10$ & $66 \pm 9$ \\
\hline Female, $n(\%)$ & $472(43)$ & $462(42)$ & $13(54)$ \\
\hline \multicolumn{4}{|l|}{ Race, $n(\%)$} \\
\hline White & $848(77)$ & $846(78)$ & $15(63)$ \\
\hline Black & $176(16)$ & $185(17)$ & $90(37)$ \\
\hline Other & $73(7)$ & $57(5)$ & $0(0)$ \\
\hline eGFR, mL/min $/ 1.73 \mathrm{~m}^{2}($ mean \pm SD) & $22.5 \pm 4.6$ & $22.4 \pm 4.3$ & $29.4 \pm 6.9$ \\
\hline UACR, mg/g (geometric mean) & 221 & 210 & 159 \\
\hline Serum magnesium, $\mathrm{mEq} / \mathrm{L}($ mean $\pm \mathrm{SD})$ & $1.72 \pm 0.23$ & $1.71 \pm 0.23$ & $1.86 \pm 0.16$ \\
\hline
\end{tabular}

${ }^{\text {a }}$ Partially reproduced from De Zeeuw et al. [17] (copyright (C) 2013).

publications also describe the demographics and baseline characteristics of the patients randomized to bardoxolone methyl versus those randomized to placebo in BEACON [17], which are also summarized in Table 1.

Pharmacodynamic Study

Twenty-four patients were enrolled in the pharmacodynamic study, and their baseline characteristics are listed in Table 1. Because the study was terminated early, only 15 (63\%) patients received all planned dose administrations and only 10 patients completed all study assessments according to the protocol.

\section{Effects of Bardoxolone Methyl on Serum Magnesium}

The baseline serum magnesium levels in patients randomized to bardoxolone methyl were similar to those randomized to placebo (mean $\pm \mathrm{SD}: 1.71 \pm 0.23$ vs. $1.72 \pm 0.23 \mathrm{mEq} / \mathrm{L}$, respectively). Changes in serum magnesium levels over time for patients randomized to bardoxolone methyl or placebo in BEACON are shown in Figure 1. The decreases in serum magnesium levels with bardoxolone methyl were apparent by week 4 and plateaued after weeks 8 to 12. Nevertheless, patients randomized to bardoxolone methyl had significant decreases in serum magnesium compared to placebo $(-0.17 \mathrm{mEq} / \mathrm{L}, 95 \% \mathrm{CI}-0.18$ to -0.60 $\mathrm{mEq} / \mathrm{L} ; p<0.001$ ) [17].

Patients in BEACON also participated in a 4-week withdrawal period following the last on-treatment visit. Four weeks after the last dose, the mean serum magnesium levels in patients randomized to bardoxolone methyl were similar to baseline (mean \pm SD: $1.69 \pm 0.26$ $\mathrm{mEq} / \mathrm{L} ; n=864$ ) and placebo patients (mean \pm SD: $1.72 \pm 0.25 \mathrm{mEq} / \mathrm{L} ; n=896$ ).

Similar effects were observed in the pharmacodynamic study (Fig. 2). Serum magnesium concentrations decreased significantly from baseline $(p<0.01)$ on day 28 and remained significantly lower through the end of treatment. Four weeks after the last dose (day 84), serum magnesium concentrations were not statistically significantly different than at baseline $(p>0.05)$.

\section{Effects of Bardoxolone Methyl on Intracellular and Urinary Magnesium}

Mean (SEM) percent change from baseline in red blood cells, sublingual epithelial cells, and 24-hour urinary magnesium levels from the pharmacodynamic study are also plotted in 
Rizk et al.: Effects of Bardoxolone Methyl on Magnesium

Fig. 1. Changes from baseline in serum magnesium in BEACON. Mean changes $( \pm$ SEM) from baseline in serum magnesium ( $\mathrm{mEq} / \mathrm{L})$ for patients randomized to bardoxolone methyl or placebo over time.

Fig. 2. Mean percent change from baseline in serum, intracellular, and urinary magnesium with bardoxolone methyl in the pharmacodynamic study. Results from an open-label pharmacodynamic study in patients $(n=24)$ with T2DM and stage 3b-4 CKD. Data plotted are mean \pm SE percent changes from baseline in serum (black), red blood cell (red), sublingual epithelial cell (green), and urine (blue) magnesium levels in patients administered bardoxolone methyl (through study day 56) and post-treatment (study day 84 ). ${ }^{* *} p<0.01$ vs. baseline. Values are plotted as mean \pm SEM at baseline $(n=24)$, study day 24 $(n=21)$, and study day $56(n=$ 20).

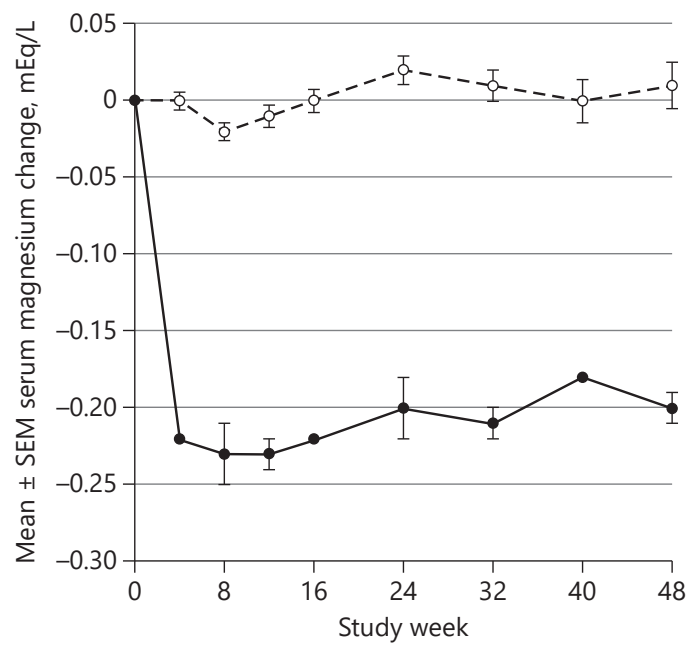

- Placebo

- Bardoxolone methyl $n=1,088$

$n=729$

$n=282$

$n=627$

$n=240$

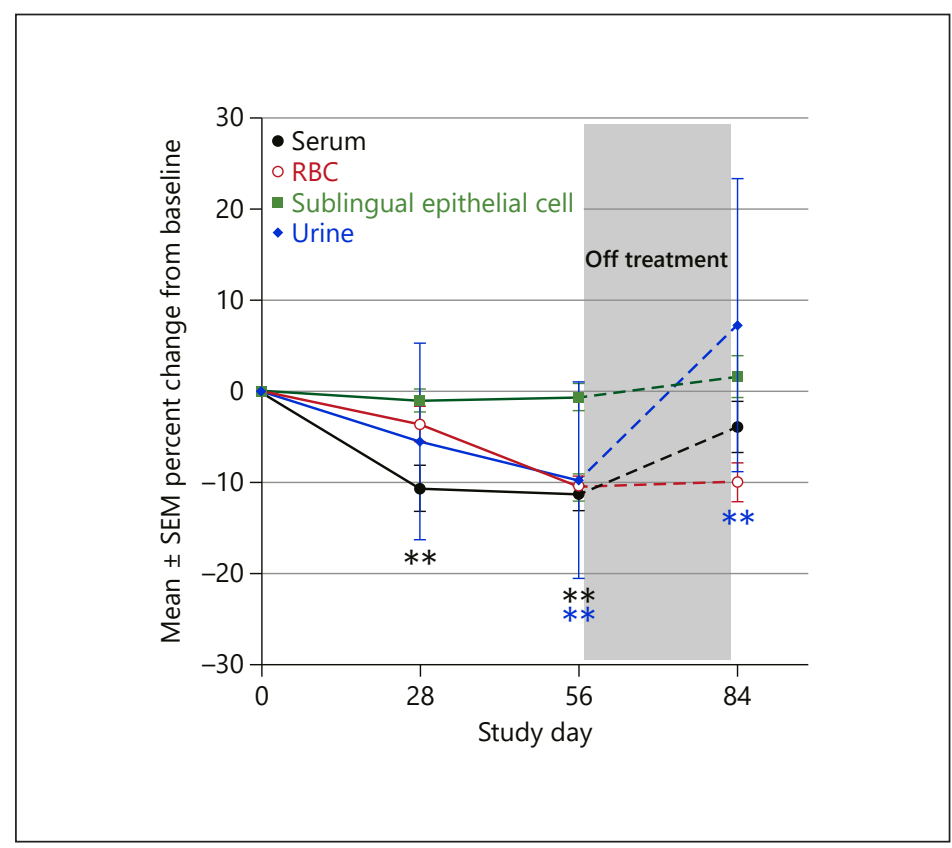

Figure 2. Magnesium concentrations in red blood cells showed a statistically significant $(p<$ 0.01 ) decrease from baseline and had a trajectory similar to serum magnesium levels, with decreases persisting through day 56 , after which concentrations generally trended back towards baseline. Notably, these changes were not accompanied by significant changes in intracellular magnesium levels, since mean magnesium concentrations in sublingual epithelial cells (which correlate with intracellular cardiac concentrations [22]) were unchanged (mean \pm SD change of $-0.4 \pm 1.9$ and $-0.3 \pm 1.9 \mathrm{mEq} / \mathrm{L}$ at days 28 and 56, respectively; Fig. 2). Similarly, there were no significant increases in urinary magnesium excretion (mean \pm SD change of $-0.47 \pm 1.32$ and $-0.48 \pm 1.30 \mathrm{mmol} / 24 \mathrm{~h}$ at days 28 and 56, respectively; Fig. 2), fractional excretion of urinary magnesium, or creatinine excretion (Table 2). 
Table 2. Select 24-hour urinary parameters with bardoxolone methyl in the pharmacodynamic study

\begin{tabular}{|c|c|c|c|c|}
\hline & Baseline & Day 28 & Day 56 & $\begin{array}{l}\text { Day } 84 \\
\text { (off-treatment) }\end{array}$ \\
\hline \multicolumn{5}{|c|}{ 24-hour urinary creatinine, $\mathrm{mmol} / 24 \mathrm{~h}$} \\
\hline Mean (SD) & $11.2(4.4)$ & $10.8(4.2)$ & $8.9(3.5)$ & $9.0(2.8)$ \\
\hline Median & 10.3 & 9.8 & 8.9 & 8.1 \\
\hline \multicolumn{5}{|c|}{ 24-hour urinary magnesium, $\mathrm{mmol} / 24 \mathrm{~h}$} \\
\hline$N$ & 24 & 19 & 14 & 9 \\
\hline Mean (SD) & $3.19(1.73)$ & $2.75(1.27)$ & $2.93(1.94)$ & $3.32(1.63)$ \\
\hline Median & 2.84 & 2.70 & 2.68 & 3.00 \\
\hline \multicolumn{5}{|c|}{ Fractional excretion of magnesium, \% } \\
\hline$N$ & 24 & 19 & 14 & 9 \\
\hline Mean (SD) & $10(4)$ & $8(4)$ & $9(4)$ & $10(5)$ \\
\hline Median & 9 & 8 & 9 & 9 \\
\hline
\end{tabular}

Table 3. QTc Fridericia interval in BEACON

\begin{tabular}{|c|c|c|c|c|}
\hline & \multicolumn{2}{|l|}{ Observed } & \multicolumn{2}{|c|}{ Change from baseline } \\
\hline & $\begin{array}{l}\text { placebo } \\
(n=1,097)\end{array}$ & $\begin{array}{l}\text { bardoxolone methyl } \\
(n=1,088)\end{array}$ & $\begin{array}{l}\text { placebo } \\
(n=1,097)\end{array}$ & $\begin{array}{l}\text { bardoxolone methyl } \\
(n=1,088)\end{array}$ \\
\hline \multicolumn{5}{|l|}{ Baseline } \\
\hline$n$ & 1,088 & 1,075 & - & - \\
\hline Mean (SD), ms & $425.1(28.0)$ & $427.4(28.9)$ & - & - \\
\hline \multicolumn{5}{|l|}{ Week 24} \\
\hline$n$ & 722 & 636 & 722 & 634 \\
\hline Mean (SD), ms & $428.8(29.1)$ & $425.9(26.5)$ & $3.6(16.3)^{\mathrm{a}}$ & $-0.9(19.2)^{b}$ \\
\hline \multicolumn{5}{|l|}{ Week 48} \\
\hline$n$ & 278 & 239 & 278 & 238 \\
\hline Mean (SD), ms & 430.7 (28.8) & $426.6(26.4)$ & $6.2(18.5)^{a}$ & $-1.7(20.2)^{\mathrm{b}}$ \\
\hline
\end{tabular}

${ }^{\mathrm{a}} p$ value $<0.01$ versus baseline. ${ }^{\mathrm{b}} p$ value $<0.01$ for bardoxolone methyl versus placebo patients.

\section{Effects of Bardoxolone Methyl on QTCF}

The mean $( \pm \mathrm{SD})$ baseline $\mathrm{QTcF}$ values in patients randomized to bardoxolone methyl or placebo in BEACON were $427.4 \pm 28.9$ and $425.1 \pm 28.0 \mathrm{~ms}$, respectively. Table 3 shows the observed QTcF values and changes from baseline in QTcF at weeks 24 and 48 in BEACON. After 24 and 48 weeks of treatment, patients randomized to placebo had significant increases from baseline in QTcF. In contrast, bardoxolone methyl patients had numerical decreases from baseline in QTcF that were significantly different relative to QTcF changes in placebo patients. Similarly, in a separate cardiovascular safety ("thorough QT") study, treatment with 20-mg or 80-mg doses of bardoxolone methyl were associated with small, dose-dependent, decreases from baseline in QTcF. Importantly, the upper limits of the 2-sided 90\% CIs for placebo-corrected changes from baseline in QTcF demonstrated that bardoxolone methyl does not prolong the QTc interval at doses as high as 80 mg. 


\section{Discussion}

In BEACON, bardoxolone methyl treatment resulted in small but statistically significant reductions in serum magnesium levels relative to baseline and placebo. The decreases in serum magnesium were attenuated after 8-12 weeks of treatment, and the decreases were reversible, with serum magnesium concentrations returning to baseline after cessation of the study drug. Similar effects on serum magnesium were observed in an open-label pharmacodynamics study as well as a prior phase 2 trial (BEAM) in patients with CKD.

Only $0.3 \%$ of total body magnesium is found in serum [23], so decreases in serum magnesium do not necessarily reflect total body magnesium levels. Importantly, urinary and intracellular magnesium levels were unchanged in a separate pharmacodynamic study. These data support the notion that reductions in serum magnesium observed with bardoxolone methyl treatment in CKD patients are not associated with renal magnesium wasting or total body magnesium depletion. Consistent with this finding, no changes in other electrolytes, including potassium and calcium (online suppl. Appendix; for all online suppl. material, see www.karger.com/doi/10.1159/000500612), were observed, suggesting that the reductions in serum magnesium were not due to tubular toxicity.

Approximately two-thirds of patients enrolled in BEACON were receiving concomitant diuretics at baseline $(64 \%$ of placebo patients [702/1,097] and $63.5 \%$ of bardoxolone methyl patients [691/1,088]) [17]. We did not control for diuretic use in the present analyses; nevertheless, if diuretics were responsible for urinary loss of magnesium, we would expect to see increases in urinary magnesium with bardoxolone methyl treatment. Instead, in the pharmacodynamic study, urinary magnesium levels actually decrease, suggesting that the decreases in serum magnesium are unlikely related to diuretic use.

Although low magnesium levels can increase the risk for arrhythmias, there was no association between decreases in serum magnesium and prolonged QT interval with bardoxolone methyl treatment. Rather, patients randomized to bardoxolone methyl in BEACON had decreases in QTcF at weeks 24 and 48 relative to baseline and relative to placebo. Importantly, as shown in a separate thorough QT cardiovascular safety study, bardoxolone methyl treatment did not increase placebo-corrected QTcF $(\Delta \Delta \mathrm{QTcF})$ at doses as high as $80 \mathrm{mg}$. Thus, decreases in serum magnesium with bardoxolone methyl are not associated with QTcF increases.

Muscle spasms were the most frequently reported adverse event in BEACON, occurring in $42 \%$ of bardoxolone methyl patients $(460 / 1,088)$ and $15 \%$ of placebo patients $(169 / 1,097)$, and in $50 \%$ of patients in the pharmacodynamic study $(12 / 24)$. The present study showed that 24-hour urinary creatinine excretion was not increased (Table 2) and prominent laboratory findings from other studies have shown that lactate dehydrogenase, BUN, uric acid, phosphorus, and potassium levels were also not increased in bardoxolone methyl subjects and therefore support the notion that muscle spasms are not associated with muscle toxicity. Instead, muscle spasms may result from improved insulin sensitivity and glucose uptake. Notably, concurrent with serum magnesium reductions, a trend of decreasing mean serum creatine phosphokinase levels were observed in BEACON and the pharmacodynamic study (online suppl. Appendix), which are inconsistent with muscle injury. Moreover, since magnesium is a cofactor for creatine phosphokinase in the conversion from adenosine triphosphate to adenosine diphosphate [23], the decreases in serum magnesium may occur due to an intracellular shift of magnesium to higher energy-demanding cells, such as muscle cells, and therefore reflect improved muscle metabolism. Analyses from prior studies with bardoxolone methyl have also shown that decreases in magnesium are not associated with the incidence of reported muscle spasm or gastrointestinal (e.g., diarrhea, vomiting) adverse events $[16,24]$. 
In summary, in addition to improvements in kidney function, treatment with bardoxolone methyl is associated with mild decreases in serum magnesium levels. The combination of decreased serum magnesium levels, together with unchanged intracellular magnesium in epithelial cells and no evidence of external loss of magnesium (e.g., no increase in urinary magnesium), suggests an intracellular shift of magnesium caused by bardoxolone methyl. Importantly, data from BEACON, an open-label pharmacodynamic study, and a cardiovascular safety study support the notion that these decreases are not likely due to adverse kidney effects (e.g., renal magnesium wasting or tubular toxicity) and are not associated with adverse cardiovascular effects on QT interval. Instead, the durable increases in eGFR with bardoxolone methyl have been shown to significantly reduce the likelihood of kidney failure outcomes, including end-stage renal disease and other validated surrogates ( $\geq 30 \%$ decline in eGFR or eGFR $<15 \mathrm{~mL} / \mathrm{min} / 1.73 \mathrm{~m}^{2}$ ) [25].

The strengths of the BEACON trial include a randomized design, a diverse patient population, and high clinical relevance to patients with T2DM and CKD. Because the pharmacodynamic study was exploratory in nature, the sample size study was selected based on practical considerations to evaluate the pharmacokinetics of bardoxolone methyl in patients, which involved intensive study visits with numerous blood draws. As a result, limitations of these analyses include the small sample size in the pharmacodynamic study, and the number of patients who completed the full 56-day dosing period was further reduced because of early termination of the study. In addition, we did not control for factors such as dietary intake of magnesium, use of diuretics or proton pump inhibitors, and diarrhea or other intestinal losses of magnesium in BEACON or the pharmacodynamic study, which can also affect magnesium levels. Furthermore, the trial did not include assessments specifically designed to determine how bardoxolone methyl affects intracellular magnesium in other cell types, including muscle cells or myocardial cells.

Further studies are needed to understand the potential mechanisms by which bardoxolone methyl might cause transcellular shifts of magnesium.

Bardoxolone methyl is currently being studied in a global phase $2 / 3$ study in patients with CKD due to Alport syndrome (CARDINAL; NCT03019185); in a phase 2 study in US patients with other rare forms of CKD, including autosomal dominant polycystic kidney disease, type 1 diabetes, IgA nephropathy, and focal segmental glomerulosclerosis (PHOENIX, NCT03366337); and in a phase 3 study in Japanese patients with type 2 diabetes and CKD (AYAME, NCT03550443).

\section{Acknowledgements}

We acknowledge the supportive role of all investigators, support staff, and patients.

\section{Statement of Ethics}

The study was approved by the Institutional Review Board at the participating site and was conducted in accordance with the Declaration of Helsinki. All patients or subjects provided written informed consent.

\section{Disclosure Statement}

D.V.R. has received research funding from Reata Pharmaceuticals, FAST biomedical and Genentech, Retrophin Inc., and Achilion Pharmaceuticals.

A.L.S. receives research funding from Reata Pharmaceuticals.

P.E.P. reports consulting fees from Reata Pharmaceuticals during the conduct of the study; his institution, Clinical Advancement Center PLLC, receives financial support because he is a PI of multiple studies. 
R.T. is a consultant for Relypsa, AstraZeneca, Amgen, Bayer, Boehringer-Ingelheim, Novo Nordisk, Reata Pharmaceuticals, and Quintiles.

D.G.W. is an investor in Reata Pharmaceuticals, and a consultant for Reata Pharmaceuticals, Sanofi/ Genzyme Corporation, Actelion Pharmaceuticals, LTD, and Protalix Biotherapeutics.

M.C., A.G., M.O., and C.M. are employees of Reata Pharmaceuticals.

P.A.M. is a consultant to Reata Pharmaceuticals.

\section{Funding Sources}

The study sponsor, Reata Pharmaceuticals, played an active role in trial design, analysis, and interpretation of data, in the writing of the report, and in the decision to submit the paper for publication.

\section{Author Contributions}

Authors had full access to all data from the trial and approved the decision to submit for publication.

\section{References}

1 Dinkova-Kostova AT, Liby KT, Stephenson KK, Holtzclaw WD, Gao X, Suh N, et al. Extremely potent triterpenoid inducers of the phase 2 response: correlations of protection against oxidant and inflammatory stress. Proc Natl Acad Sci USA. 2005 Mar;102(12):4584-9.

2 Li J, Stein TD, Johnson JA. Genetic dissection of systemic autoimmune disease in Nrf2-deficient mice. Physiol Genomics. 2004 Aug;18(3):261-72.

3 Yoh K, Itoh K, Enomoto A, Hirayama A, Yamaguchi N, Kobayashi M, et al. Nrf2-deficient female mice develop lupus-like autoimmune nephritis. Kidney Int. 2001 Oct;60(4):1343-53.

4 Ma Q, Battelli L, Hubbs AF. Multiorgan autoimmune inflammation, enhanced lymphoproliferation, and impaired homeostasis of reactive oxygen species in mice lacking the antioxidant-activated transcription factor Nrf2. Am J Pathol. 2006 Jun;168(6):1960-74.

5 Yates MS, Tauchi M, Katsuoka F, Flanders KC, Liby KT, Honda T, et al. Pharmacodynamic characterization of chemopreventive triterpenoids as exceptionally potent inducers of Nrf2-regulated genes. Mol Cancer Ther. 2007 Jan;6(1):154-62.

6 Sporn MB, Liby KT, Yore MM, Fu L, Lopchuk JM, Gribble GW. New synthetic triterpenoids: potent agents for prevention and treatment of tissue injury caused by inflammatory and oxidative stress. J Nat Prod. 2011 Mar; 74(3):537-45.

7 Ahmad R, Raina D, Meyer C, Kharbanda S, Kufe D. Triterpenoid CDDO-Me blocks the NF-kappaB pathway by direct inhibition of IKKbeta on Cys-179. J Biol Chem. 2006 Nov;281(47):35764-9.

8 Straus DS, Pascual G, Li M, Welch JS, Ricote M, Hsiang CH, et al. 15-deoxy-delta 12,14-prostaglandin J2 inhibits multiple steps in the NF-kappa B signaling pathway. Proc Natl Acad Sci USA. 2000 Apr;97(9):4844-9.

9 Serhan CN, Chiang N, Van Dyke TE. Resolving inflammation: dual anti-inflammatory and pro-resolution lipid mediators. Nat Rev Immunol. 2008 May;8(5):349-61.

10 Levonen AL, Landar A, Ramachandran A, Ceaser EK, Dickinson DA, Zanoni G, et al. Cellular mechanisms of redox cell signalling: role of cysteine modification in controlling antioxidant defences in response to electrophilic lipid oxidation products. Biochem J. 2004 Mar;378(Pt 2):373-82.

11 Wu QQ, Wang Y, Senitko M, Meyer C, Wigley WC, Ferguson DA, et al. Bardoxolone methyl (BARD) ameliorates ischemic AKI and increases expression of protective genes Nrf2, PPAR $\gamma$, and HO-1. Am J Physiol Renal Physiol. 2011 May;300(5):F1180-92.

12 Ma R, Bumeister R, Stidham R, Kambuj P, Sprouse M, Ferguson D, et al. Bardoxolone Methyl (BARD). Germany: Inhibits Inflammatory Signaling in Cultured Mesangial Cells. Poster ERA-EDTA Meeting Munich; 2010.

13 Ding Y, Stidham RD, Bumeister R, Trevino I, Winters A, Sprouse M, et al. The synthetic triterpenoid, RTA 405, increases the glomerular filtration rate and reduces angiotensin II-induced contraction of glomerular mesangial cells. Kidney Int. 2013 May;83(5):845-54.

14 Aminzadeh MA, Reisman SA, Vaziri ND, Khazaeli M, Yuan J, Meyer CJ. The synthetic triterpenoid RTA dh404 (CDDO-dhTFEA) restores Nrf2 activity and attenuates oxidative stress, inflammation, and fibrosis in rats with chronic kidney disease. Xenobiotica. 2014 Jun;44(6):570-8.

15 Pergola PE, Krauth M, Huff JW, Ferguson DA, Ruiz S, Meyer CJ, et al. Effect of bardoxolone methyl on kidney function in patients with T2D and Stage 3b-4 CKD. Am J Nephrol. 2011;33(5):469-76.

16 Pergola PE, Raskin P, Toto RD, Meyer CJ, Huff JW, Grossman EB, et al.; BEAM Study Investigators. Bardoxolone methyl and kidney function in CKD with type 2 diabetes. N Engl J Med. 2011 Jul;365(4):327-36. 
17 de Zeeuw D, Akizawa T, Audhya P, Bakris GL, Chin M, Christ-Schmidt H, et al.; BEACON Trial Investigators. Bardoxolone methyl in type 2 diabetes and stage 4 chronic kidney disease. N Engl J Med. 2013 Dec;369(26): 2492-503.

18 Nangaku M, Shimazaki R, Akizawa T. Bardoxolone Methyl Improved GFR Measured by Standard Inulin Clearance: the TSUBAKI Study. J Am Soc Nephrol. 2017;28:B1.

19 Chin MP, Reisman SA, Bakris GL, O'Grady M, Linde PG, McCullough PA, et al. Mechanisms contributing to adverse cardiovascular events in patients with type 2 diabetes mellitus and stage 4 chronic kidney disease treated with bardoxolone methyl. Am J Nephrol. 2014;39(6):499-508.

20 Chin MP, Wrolstad D, Bakris GL, Chertow GM, de Zeeuw D, Goldsberry A, et al. Risk factors for heart failure in patients with type 2 diabetes mellitus and stage 4 chronic kidney disease treated with bardoxolone methyl. J Card Fail. 2014 Dec;20(12):953-8.

21 de Zeeuw D, Akizawa T, Agarwal R, Audhya P, Bakris GL, Chin M, et al. Rationale and trial design of Bardoxolone Methyl Evaluation in Patients with Chronic Kidney Disease and Type 2 Diabetes: the Occurrence of Renal Events (BEACON). Am J Nephrol. 2013;37(3):212-22.

22 Haigney MC, Silver B, Tanglao E, Silverman HS, Hill JD, Shapiro E, et al. Noninvasive measurement of tissue magnesium and correlation with cardiac levels. Circulation. 1995 Oct;92(8):2190-7.

23 Jahnen-Dechent W, Ketteler M. Magnesium basics. Clin Kidney J. 2012 Feb; 5 Suppl 1:i3-14.

24 Chin M, Goldsberry A, Hebbar S, Meyer C, Audhya P, Toto R, et al. Bardoxolone Methyl Acutely Reduces Serum Magnesium in Stage 3b and 4 CKD Patients with Type 2 Diabetes without any Adverse Effect on QT Interval. France: Presentation ERA-EDTA Meeting Paris; 2012.

25 Chin MP, Bakris GL, Block GA, Chertow GM, Goldsberry A, Inker LA, et al. Bardoxolone Methyl Improves Kidney Function in Patients with Chronic Kidney Disease Stage 4 and Type 2 Diabetes: Post-Hoc Analyses from Bardoxolone Methyl Evaluation in Patients with Chronic Kidney Disease and Type 2 Diabetes Study. Am J Nephrol. 2018;47(1):40-7. 